\title{
Central Nervous System Tuberculosis: Clinical Characteristics and Outcome. A Saudi Tertiary Care Centre Experience
}

\author{
Mohammad Bo Saeed1* ${ }^{*}$ Adel Alothman1,2, Suleiman Kojan1,2, Suliman Almahmoud1, \\ Ali Al Khathaami1,2, Mohammed Al Ghobain ${ }^{1,2}$ \\ ${ }^{1}$ Department of Medicine, King Abdulaziz Medical City, Riyadh, Saudi Arabia \\ ${ }^{2}$ King Abdullah International Medical Research Center, King Saud bin Abdulaziz University for Health Sciences, \\ Riyadh, Saudi Arabia \\ Email: dr.bosaeed@live.com
}

Received 24 February 2015; accepted 18 March 2015; published 24 March 2015

Copyright (C) 2015 by authors and Scientific Research Publishing Inc.

This work is licensed under the Creative Commons Attribution International License (CC BY). http://creativecommons.org/licenses/by/4.0/

(c) (i) Open Access

\section{Abstract}

Background: Tuberculosis is an endemic problem that is of important public health concern in Saudi Arabia. Available recent prevalence of tuberculosis (pulmonary and extra-pulmonary) was estimated to be 13.7 per 100,000 people living in the Kingdom of Saudi Arabia. Methods: A retrospective chart review of all Central Nervous System tuberculosis patients (CNS-TB) treated at King Abdulaziz Medical City, Riyadh, Saudi Arabia between 1996 and 2010. CNS-TB was defined as follows: patients who had symptoms and signs of CNS-TB with radiographic, microbiologic, or histopathologic evidence of tuberculous infection and/or those with highly probable diagnosis, supported by radiographic typical features, not confirmed microbiologically but who responded to anti-TB therapy. Results: Eighty two patients (46 males) met our definition with the mean age of 50 years. Only 11 patients $(13.4 \%)$ reported previous TB infection. The most common presenting symptoms were: headache $(51 \%)$, fever $(50 \%)$, weakness $(43 \%)$, confusion $(29 \%)$ and seizures $(28 \%)$. The most common signs found by clinical examination were: weakness $(45 \%)$ followed by sensory impairment. Positive CSF acid-fast bacilli (AFB) culture confirmed the diagnosis in 20 out of 49 tested patients (about 41\%). Brain CT scan was done in 74 patients and meningeal enhancement was identified in only 6 patients $(8 \%)$ but ring enhancing lesions were found in 19 patients (26\%). In MRI meningeal enhancement was founded in 26 patients $(37 \%)$ and ring enhanced lesions in 36 patients $(51 \%)$. After receiving treatment, 45 patients $(55 \%)$ had complete or good recovery and 23 patients $(28 \%)$ had partial recovery. However, $15 \%$ (12 patients) had poor or no improvement. 2 patients $(2 \%)$ lost their follow up. Conclusion: CNS-TB continues to be a major health issue especially in endemic areas such as Saudi Arabia. The diverse clinical presentations, lack of high yield, practical and reliable diagnosis methods and inconsistent management

\footnotetext{
*Corresponding author.
}

How to cite this paper: Bo Saeed, M., Alothman, A., Kojan, S., Almahmoud, S., Al Khathaami, A. and Al Ghobain, M. (2015) Central Nervous System Tuberculosis: Clinical Characteristics and Outcome. A Saudi Tertiary Care Centre Experience. Advances in Infectious Diseases, 5, 63-71. http://dx.doi.org/10.4236/aid.2015.51007 
plans require further research and harder work in order to meet the current challenges and reach a unified case definition and evidence based management guidelines that would be a very practical step.

\section{Keywords}

\section{Tuberculosis, Central Nervous System, Saudi Arabia}

\section{Introduction}

Tuberculosis (TB) continues to be one of the most important communicable diseases worldwide due to significant morbidity and mortality despite modern anti-tuberculosis chemotherapy. It accounted for 1.4 Million deaths worldwide in 2012 alone [1]. Central nervous system tuberculosis (CNS-TB), most severe form of extra-pulmonary TB, constitutes of $1 \%$ of all newly diagnosed TB cases [2] and about $6 \%$ of all extra-pulmonary TB in immunocompetent individuals [3].

The pathogenesis of CNS-TB is poorly understood plus there are no rapid, sensitive and reliable diagnostic tests. In addition, delaying its diagnosis and/or treatment, often, leads to increased morbidity and mortality [4] [5] making it a very challenging and serious disorder [6].

There are no standardized diagnostic criteria for CNS-TB and the available published case series are heterogeneous since different case definitions had been adopted [7]-[14]. Plus there are no randomized, controlled trials to determine optimal drug combination, dosage, or duration of anti-tuberculous chemotherapy. The current principles of CNS-TB treatment are those that govern the management of pulmonary TB [15] [16].

In Saudi Arabia, tuberculosis is an endemic illness that is of important public health concern. Available recent prevalence in 2011 of tuberculosis (pulmonary and extra-pulmonary) was estimated to be 13.7 per 100,000 populations in Saudi Arabia [17]. Reported CNS-TB from Saudi Arabia varied widely between hospitals and at different time periods [18]. In 1982, Froude et al. found that CNS TB was the most common form in 162 patients diagnosed with extra-pulmonary TB [19]. More recently, Al-Otaibi et al. reviewed 248 patients diagnosed with extra-pulmonary TB at King Khalid University Hospital, Riyadh and CNS-TB accounted for $4.4 \%$ of extrapulmonary TB cases admitted between 2001 and 2007 [20].

\section{Methods}

A retrospective chart review of all CNS-TB patients treated at King Abdulaziz Medical City (KAMC), Riyadh, Saudi Arabia between 1996 and 2010 inclusive. KAMC is a distinguished healthcare provider in Saudi Arabia with a capacity of more than 700 beds and located in Riyadh city. The study included the patients who was admitted and followed up in this hospital.

CNS-TB was defined as follows: patients who had symptoms and signs of CNS Tuberculosis with radiographic, microbiologic, or histopathologic evidence of tuberculous infection and/or those with highly probable diagnosis, supported by radiographic typical features, not confirmed microbiologically but who responded to anti-TB therapy.

Patient's demography, presenting symptoms and signs, Cerebrospinal Fluid (CSF) parameters, radiological changes, treatment regimen, and final outcome were reviewed.

Patients' outcome was classified as: 1) full or good recovery (resumption of patient's normal life with no or minor neurological or psychological deficits); 2) partial recovery (mild to moderate disability, but independent activities of daily living); 3) poor or no improvement (dependent for activities of daily living due to moderate to severe mental or physical disability, including persistent vegetative state and death); 4) outcome status not available.

The data were entered into a Microsoft Excel spreadsheet. Statistical Analysis System SAS was used for data analysis.

The study was approved from King Abdullah International Medical Research Center at King Saud bin Abdulaziz University for Health Sciences before starting data collection as the study does not disclose patient identity, and poses no risks to patients (Reference N. RR11/054). This approval granted a permission to conduct the study 
and there was no need for informed consent from patients.

\section{Results and Discussion}

A total of 124 patients, labeled as CNS-TB, were treated at KAMC-Riyadh between 1996 and 2010. However, only 82 patients (46 males 56\%) met our case definition and the remaining 42 patients were excluded.

The mean age was 50 years. Only 11 patients (13.4\%) reported previous TB infection. About one quarter of patients had at least one chronic co-morbid disorder such as diabetes mellitus (23\%) and hypertension (26\%) (Table 1).

\subsection{Presentation}

Time to diagnosis ranged from 1 day to 10 months (mean 38 days). The most common presenting symptoms were: headache $(51 \%)$, fever (50\%), weakness (43\%), confusion (29\%) and seizures (28\%). Other symptoms such as nausea and vomiting presented in $8 \%$ and $32 \%$ respectively. $18 \%$ of patients presented with coma (Table 2).

The most common signs found by clinical examination were: weakness (45\%) followed by sensory impairment. $23 \%$ of the patients were reported to have encephalopathy and impaired level of consciousness. Documented fever upon presentation was found in $35 \%$ of patients but meningeal signs were uncommon and only found in $12 \%$ of them (Table 2).

Table 1. Characteristics of 82 patients with CNS-TB.

\begin{tabular}{|c|c|}
\hline Characteristic & $\mathrm{n}=82(\%)$ \\
\hline \multicolumn{2}{|l|}{ Age (years) } \\
\hline Range & 5 - 90 years \\
\hline Mean & 50 years \\
\hline \multicolumn{2}{|l|}{ Gender } \\
\hline Males & 46 (56\%) \\
\hline Females & $36(44 \%)$ \\
\hline Past history of TB & $11(13 \%)$ \\
\hline Pulmonary & 3 \\
\hline Spine & 2 \\
\hline Miliary & 2 \\
\hline Peritoneal & 1 \\
\hline Lymph nodes & 1 \\
\hline CNS & 2 \\
\hline \multicolumn{2}{|l|}{ Co-morbidities } \\
\hline Diabetes Mellitus & 19 (23\%) \\
\hline Hypertension & $21(26 \%)$ \\
\hline Chronic kidney disease & $9(11 \%)$ \\
\hline Chronic liver disease & $2(2 \%)$ \\
\hline Solid organ transplant & $2(2 \%)$ \\
\hline \multicolumn{2}{|l|}{ Date of presentation } \\
\hline 1996-1999 & $18(22 \%)$ \\
\hline $2000-2005$ & $34(41 \%)$ \\
\hline $2006-2010$ & $30(37 \%)$ \\
\hline
\end{tabular}


Table 2. Clinical features of CNS tuberculosis patients.

\begin{tabular}{|c|c|}
\hline Symptoms & $\mathrm{n}=82(\%)$ \\
\hline \multicolumn{2}{|l|}{-Constitutional: } \\
\hline - Fever & $41(50 \%)$ \\
\hline - Weight loss & $13(16 \%)$ \\
\hline - Sweating & $10(12 \%)$ \\
\hline - Fatigue & $7(8 \%)$ \\
\hline \multicolumn{2}{|l|}{-Specific: } \\
\hline - Headache & $42(51 \%)$ \\
\hline - Weakness & 35 (43\%) \\
\hline - Seizure & $23(28 \%)$ \\
\hline - Confusion & $25(30 \%)$ \\
\hline - Urine incontinence & $5(6 \%)$ \\
\hline - Dizziness & $6(7 \%)$ \\
\hline - Visual disturbance & $4(5 \%)$ \\
\hline - Photophobia & $3(3 \%)$ \\
\hline - Coma & $15(18 \%)$ \\
\hline \multicolumn{2}{|l|}{-Others: } \\
\hline - Vomiting & $26(32 \%)$ \\
\hline - Anorexia & $15(18 \%)$ \\
\hline - Nausea & 7 (8\%) \\
\hline \multicolumn{2}{|l|}{ Signs: } \\
\hline - Fever & $29(35 \%)$ \\
\hline - Lymphadenopathy & $3(3 \%)$ \\
\hline - Impaired conscious level & $19(23 \%)$ \\
\hline - Disorientation & 8 (9\%) \\
\hline - Meningeal signs & $10(12 \%)$ \\
\hline - Neurological deficit & 37 (45\%) \\
\hline Sensory & $7(8 \%)$ \\
\hline Motor & 37 (45\%) \\
\hline - Ataxia & $3(3 \%)$ \\
\hline
\end{tabular}

\subsection{Investigations}

Tuberculin skin test was not performed routinely for our patients.

Lumbar puncture (LP) was performed, on the day of admission, in 59 patients (72\%) (Table 3). The remaining patients were denied LP due increased procedure risk (symptoms and/or signs of increased intra-cranial pressure) or had alternative working diagnoses during the first admission day. The CSF white cell count (WBC) was elevated in 48 out of 59 tested patients (81\%) with no specific differentials. Total CSF protein was elevated 
Table 3. Cerebrospinal fluid characteristics.

\begin{tabular}{cc}
\hline CSF protein $\mathbf{g} \mathbf{L}$ & $\mathbf{n}=\mathbf{5 5}(\mathbf{\%})$ \\
\hline High $(>0.40)$ & $46(83 \%)$ \\
Normal $(0.15-0.40)$ & $7(13 \%)$ \\
Low $(<0.15)$ & $2(4 \%)$ \\
\hline CSF: serum glucose ratio & $\mathbf{n = 5 3 ( \% )}$ \\
\hline Less than 0.3 & $1936 \%$ \\
\hline $0.3-0.6$ & $2445 \%$ \\
\hline More than 0.6 & $1019 \%$ \\
\hline CSF AFB & $\mathbf{n}=\mathbf{4 9}(\mathbf{\%})$ \\
\hline Smear positive & $2(4 \%)$ \\
\hline Culture positive & $20(41 \%)$ \\
\hline
\end{tabular}

in 46 out of 55 tested patients (83\%). Very high protein level (above $3 \mathrm{~g} / \mathrm{L}$ ) was noticed in about $25 \%$ (14 patients). 33\% (18 patients) had CSF glucose below the minimum reference value. The CSF to serum glucose ratio could be calculated in a total of 53 patients. The ratio was below 0.5 in $66 \%$ of these patients.

Only 2 patients had positive AFB smear in the CSF. Positive CSF Mycobacterium Tuberculosis MTB culture confirmed the diagnosis in 20 out of 49 tested patients (about 41\%). Drug susceptibility testing for first line drugs was performed for all culture positive cases. Only one isolate (5\%) was resistant to first line therapy (both Rifampicin and Isoniazid).

Other non-specific laboratory features such as leukocytosis (WBC > $11.0 \times 10^{9} /$ Liter), hyponatremia (sodium level below $135 \mathrm{mmol} / \mathrm{L}$ ), were found in $23 \%$ and 52\% respectively. In about $30 \%$ of the patients the serum sodium was significantly reduced (less than $130 \mathrm{mmol} / \mathrm{L}$ ). Chest X-ray was performed in 76 patients and was found abnormal (mostly non-specific changes) in 27 cases (36\%).

18 patients underwent meningeal and/or brain biopsies, which showed "Necrotizing Caseating Granuloma" in 11 patients (61\%), non-specific inflammation in $11 \%$ and normal results in $28 \%$. Biopsy tissues AFB stains were positive in 3 samples (17\%) and MTB culture in 2 samples (11\%).

Majority of patients had either cranial computed tomography (CT) or Magnetic resonance imaging (MRI). 63 patients had both scans performed during the same hospital stay. Brain CT scan was done in 74 patients and meningeal enhancement was identified in only 6 patients (8\%) but ring enhancing lesions were found in 19 patients (26\%). Tuberculoma like lesions were reported on $9 \%$ of the patients. Other findings such as hydrocephalus and brain edema were found in 28\% and 38\% respectively (Table 4). CT scan was normal in 13 patients, 9 of them had brain MRI performed, that had positive, additional diagnostic value by showing meningeal enhancement and/or ring enhancing lesions.

Brain MRI was done in 71 patients. $14 \%$ of the patients had tuberculomas, $17 \%$ hydrocephalus and $35 \%$ had brain edema. Meningeal enhancement was found in 26 patients (37\%) and ring-enhanced lesions in 36 patients (51\%) (Table 4).

Only two patients had both CT and MR scans normal.

\subsection{Treatment}

Standard treatment regimen as per the recommendations of American and British Thoracic Societies, Infectious Disease Society of America, and the U.S. Centers for Disease Control and Prevention was applied [15] [16]. Patients received an initial two month course of intensive therapy using the four first line therapies (Isoniazid, Rifampicin, Pyrazinamide, and Ethambutol) followed by a prolonged course lasting 7 - 10 months, of 2 drugs (Isoniazid and Rifampicin) based on clinical response and established drug sensitivity of the isolate.

Therefore almost all the patients received Isoniazid (INH) and Rifampicin initially but only $70 \%$ continued these 2 medications for full course therapy. Ethambutol and Pyrazinamide were used in $88 \%$ and $94 \%$ respectively. The first line therapy was stopped due to significant adverse effects (e.g. Drug induced hepatitis occurred 
Table 4. Neuroradiological findings of patients where both MRI \& CT was performed.

\begin{tabular}{|c|c|c|}
\hline \multicolumn{3}{|c|}{ WITHOUT contrast $\mathrm{n}=63(\%)$} \\
\hline Findings & $\underline{\text { CT scan }}$ & $\underline{\text { MRI }}$ \\
\hline Tubercoloma & $4(6 \%)$ & $7(11 \%)$ \\
\hline Hydrocephalus & $18(28 \%)$ & $12(19 \%)$ \\
\hline Edema & $23(36 \%)$ & $24(38 \%)$ \\
\hline Stroke & $7(11 \%)$ & $7(11 \%)$ \\
\hline \multicolumn{3}{|c|}{ WITH contrast $\mathrm{n}=54(\%)$} \\
\hline Meningeal enhancement & $6(11 \%)$ & $20(37 \%)$ \\
\hline Ring enhancing lesions & $14(26 \%)$ & $30(55 \%)$ \\
\hline Non-ring enhancing lesions & 17 (31\%) & 12 (22\%) \\
\hline
\end{tabular}

in 18 patients (22\%)), or lack of response or resistance. Alternative medications used included Ciprofloxacin in 10 patients and Moxifloxacin in 13. Amikacin was added to the regimen used to treat the single patient with multi-drug resistant TB. Corticosteroids were used in 62 patients (76\%).

\subsection{Clinical Outcome}

Average hospital length of stay was 39 days. About a third of patients required Intensive Care Unit (ICU) admission with an average of 20 days spent in the ICU (Table 5).

Post discharge patients were followed in the outpatient clinics for at least 12 months. 68 patients (83\%) had favorable outcome. 45 patients (55\%) had complete or good recovery and 23 patients (28\%) had partial recovery. However, 12 patients (15\%) had poor or no improvement. 2 patients (2\%) lost their follow up. 30 day mortality rate was $8.5 \%$ (7 patients had died).

\section{Discussion}

Early recognition, diagnosis and treatment are essential. However, CNS TB remains a very challenging infection due to variable, often non-specific, symptoms and signs, prolonged symptoms prior to presentation (from days to months) and lack of time efficient specific testing [21] [22]. Tuberculosis of the CNS showed variable results in diagnosis measures, management response, and prognosis comparing to other sites of the infection (e.g. spine).

Our case definition of CNS TB was based on previous studies in which similar case definition was used [23]. Still there is no recognized international CNS TB case definition but a trial of making a consensus case definition has been done based on experts meeting few years ago [24]. The different case definitions used in various studies make the comparison of research findings somewhat difficult but in general our study showed different data in regarding of the disease behavior and outcome comparing to other limited local results [18]-[20].

Available investigations, although often helpful, are time consuming plus there is no single test modality that is highly sensitive and/or specific. The gold standard diagnostic test is to culture the MTB from the CSF or the tissue that may take longer time that would significantly delay the diagnosis confirmation and starting treatment. AFB smear positivity would have saved significant amount of time and/or resources but this was rarely positive (4\%) "Section 3.2". Brain and/or meningeal biopsy, an invasive potentially harmful procedure, helped confirming the diagnosis in a number of patients. Other tests, i.e. CSF leukocyte count, total protein concentration, glucose ratio and serum sodium levels are neither highly sensitive nor specific.

Available imaging modalities are helpful to make the diagnosis and monitor therapy response. As in "Section 3.2", MRI compared to CT scan, proved more sensitive for identifying meningeal enhancement (37\% vs. $11 \%$ respectively) and ring enhancing lesions ( $55 \%$ vs. $26 \%$ respectively). Although some experts have suggested outcome predictor value of brain imaging at the time of diagnosis particularly with CT modality, however, these were not reliable or validated. Especially in those who develop imaging predictors particularly by CT images [25]-[27]. 
Table 5. Outcome of 82 patients with CNS tuberculosis.

$\mathbf{n}=\mathbf{8 2}(\%)$

\begin{tabular}{cc} 
Hospital stay & $\mathbf{n}=\mathbf{8 2}$ (\%) \\
Range & \\
Mean & 4 days - 17 months \\
ICU admission & 39 days \\
Rang & 27 (33\%) \\
Mean & 1 day - 3 months \\
Response to Anti-TB & 20 days \\
Full recovery & \\
Partial recovery & 45 (55\%) \\
No improvement & $23(28 \%)$ \\
Lost follow up & $12(15 \%)$ \\
30-day mortality & $2(2 \%)$ \\
\hline
\end{tabular}

Tuberculosis infection had been fatal until specific anti-TB drugs were discovered in the second half of the 20th century. However, both mortality and morbidity continue to be high reaching $60 \%$ particularly in developing countries. Complications can be seen in $25 \%$ of survivors despite five major and numerous minor drug options available [12]-[28]. A delay in starting anti-tuberculosis medications is associated with a higher rate of mortality and serious neurological complications in survivors. In the absence of a rapid and highly sensitive test, decision to initiate treatment is often made before the diagnosis is been confirmed.

In comparing of the disease outcome, the mortality rate in our cases was significantly lower than other reported case series and $83 \%$ of our patients had a favorable outcome [29]-[31].

It should be noted that in our study has a limitation of being only descriptive of an original data. It is retrospective chart review with some limitation of reaching all the desired information. No logistic regression analysis or advanced statistical tests were done. Further prospective studies and clinical trials are required for getting closer to understand the specifics of this disease.

\section{Conclusion}

Central nervous system tuberculosis continues to be a major health issue especially in endemic areas such as Saudi Arabia. The diverse clinical presentations, lack of high yield, practical and reliable diagnosis methods and inconsistent management plans require further research and harder work in order to meet the current challenges and reach a unified case definition and evidence based management guidelines that would be a very practical step.

\section{Competing Interests}

The authors declare that they have no competing interests.

\section{References}

[1] World Health Organization (2012) Global Tuberculosis Database. www.who.int/tb/publications/global_report/en/index.html

[2] Phypers, M., Harris, T. and Power, C. (2006) CNS Tuberculosis: A Longitudinal Analysis of Epidemiological and Clinical Features. International Journal of Tuberculosis and Lung Disease, 10, 99-103.

[3] CDC (2012) Reported Tuberculosis in the United States, 2011. US Department of Health and Human Services, Atlanta.

[4] Hosoglu, S., Geyik, M.F., Balik, I., et al. (2002) Predictors of Outcome in Patients with Tuberculous Meningitis. In- 
ternational Journal of Tuberculosis and Lung Disease, 6, 64-70.

[5] Thwaites, G., Chau, T.T.H., Mai, N.T.H., et al. (2000) Tuberculous Meningitis. Journal of Neurology, Neurosurgery \& Psychiatry, 68, 289-299. http://dx.doi.org/10.1136/jnnp.68.3.289

[6] Thwaites, G.E. and Tran, T.H. (2005) Tuberculous Meningitis: Many Questions, Too Few Answers. Lancet Neurology, 4, 160-170. http://dx.doi.org/10.1016/S1474-4422(05)70019-3

[7] Marais, S., Thwaites, G., Schoeman, J.F., Torok, M.E., Misra, U.K., et al. (2010) Tuberculous Meningitis: A Uniform Case Definition for Use in Clinical Research. The Lancet Infectious Diseases, 10, 803-812. http://dx.doi.org/10.1016/S1473-3099(10)70138-9

[8] van Well, G.T., Paes, B.F., Terwee, C.B., et al. (2009) Twenty Years of Pediatric Tuberculous Meningitis: A Retrospective Cohort Study in the Western Cape of South Africa. Pediatrics, 123, e1-e8. http://dx.doi.org/10.1542/peds.2008-1353

[9] Karande, S., Gupta, V., Kulkarni, M. and Joshi, A. (2005) Prognostic Clinical Variables in Childhood Tuberculous Meningitis: An Experience from Mumbai, India. Neurology India, 53, 191-195. http://dx.doi.org/10.4103/0028-3886.16407

[10] Saitoh, A., Pong, A., Waecker Jr., N.J., Leake, J.A., Nespeca, M.P. and Bradley, J.S. (2005) Prediction of Neurologic Sequelae in Childhood Tuberculous Meningitis: A Review of 20 Cases and Proposal of a Novel Scoring System. The Pediatric Infectious Disease Journal, 24, 207-212. http://dx.doi.org/10.1097/01.inf.0000154321.61866.2d

[11] Kalita, J., Misra, U.K. and Ranjan, P. (2007) Predictors of Long-Term Neurological Sequelae of Tuberculous Meningitis: A Multivariate Analysis. European Journal of Neurology, 14, 33-37. http://dx.doi.org/10.1111/j.1468-1331.2006.01534.x

[12] Thwaites, G.E., Bang, N.D., Dung, N.H., Thi Quy, H., Oanh, D.T.T., Cam Thoa, N.T., et al. (2004) Dexamethasone for the Treatment of Tuberculous Meningitis in Adolescents and Adults. New England Journal of Medicine, 351, 17411751. http://dx.doi.org/10.1056/NEJMoa040573

[13] van der Weert, E.M., Hartgers, N.M., Schaaf, H.S., Eley, B.S., Pitcher, R.D., Wieselthaler, N.A., et al. (2006) Comparison of Diagnostic Criteria of Tuberculous Meningitis in Human Immunodeficiency Virus-Infected and Uninfected Children. Pediatric Infectious Disease Journal, 25, 65-69. http://dx.doi.org/10.1097/01.inf.0000183751.75880.f8

[14] Torok, M.E., Chau, T.T., Mai, P.P., Duy Phong, N., Thi Dung, N., Van Chuong, L., et al. (2008) Clinical and Microbiological Features of HIV-Associated Tuberculous Meningitis in Vietnamese Adults. PLoS ONE, 3, e1772.

[15] Joint Tuberculosis Committee of the British Thoracic Society (1998) Chemotherapy and Management of Tuberculosis in the United Kingdom: Recommendations 1998. Thorax, 53, 536-548.

[16] Blumberg, H.M., Burman, W.J., Chaisson, R.E., et al. (2003) American Thoracic Society/Centers for Disease Control and Prevention/Infectious Diseases Society of America: Treatment of Tuberculosis. American Journal of Respiratory and Critical Care Medicine, 167, 603-662. http://dx.doi.org/10.1164/rccm.167.4.603

[17] Ministry of Health KSA (2011) Health Statistical Year Book. www.moh.gov.sa

[18] Alrajhi, A.A. and Al-Barrak, A.M. (2002) Extrapulmonary Tuberculosis, Epidemiology and Patterns in Saudi Arabia. Saudi Medical Journal, 23, 503-508.

[19] Froude, J.R. and Kingston, M. (1982) Extrapulmonary Tuberculosis in Saudi Arabia, a Review of 162 Cases. King Faisal Specialized Hospital Medical Journal, 2, 85-95.

[20] Al-Otaibi, F. and El Hazmi, M.M. (2010) Extra-Pulmonary Tuberculosis in Saudi Arabia. Indian Journal of Pathology and Microbiology, 53, 227-231. http://dx.doi.org/10.4103/0377-4929.64327

[21] Kennedy, D.H. and Fallon, R.J. (1979) Tuberculous Meningitis. JAMA, 241, 264-268. http://dx.doi.org/10.1001/jama.1979.03290290032021

[22] Thwaites, G.E., Chau, T.T.H., Stepniewska, K., Phu, N.H., Chuong, L.V., Sinh, D.X., et al. (2002) Diagnosis of Adult Tuberculous Meningitis by Use of Clinical and Laboratory Features. Lancet, 360, 1287-1292. http://dx.doi.org/10.1016/S0140-6736(02)11318-3

[23] Alothman, A., Memish, Z.A., Awada, A., Al Mahmood, S., Al Sadoon, S., Rahman, M.M. and Khan, M.Y. (2001) Tuberculous Spondylitis: Analysis of 69 Cases from Saudi Arabia. Spine, 26, 565-570. http://dx.doi.org/10.1097/00007632-200112150-00020

[24] Marais, S., Thwaites, G., Schoeman, J.F., Török, M.E., Misra, U.K., Prasad, K., et al. (2010) Tuberculous Meningitis: A Uniform Case Definition for Use in Clinical Research. Lancet Infectious Diseases, 10, 803-812. http://dx.doi.org/10.1016/S1473-3099(10)70138-9

[25] Toeh, R., Humphries, M.J., Hoare, R.D. and O’Mahony, G. (1989) Clinical Correlation of CT Changes in 64 Chinese Patients with Tuberculous Meningitis. Journal of Neurology, 236, 48-51. http://dx.doi.org/10.1007/BF00314218

[26] Jie, L. (1988) Computerized Tomography in Tuberculous Meningitis. Chinese Medical Journal, 101, 388-390. 
[27] Trautmann, M., Kluge, W., Otto, H.S. and Loddenkemper, R. (1986) Computed Tomography in CNS Tuberculosis. European Neurology, 25, 91-97. http://dx.doi.org/10.1159/000115993

[28] Kent, S.J., Crowe, S.M., Yung, A., Lucas, C.R. and Mijch, A.M. (1993) Tuberculous Meningitis: A 30-Year Review. Clinical Infectious Diseases, 17, 987-994. http://dx.doi.org/10.1093/clinids/17.6.987

[29] Sütlaş, P.N., Unal, A., Forta, H., Senol, S. and Kirbaş, D. (2003) Tuberculous Meningitis in Adults: Review of 61 Cases. Infection, 31, 387-391.

[30] Christensen, A.S.H., Andersen, Å.B., Thomsen, V.Ø., Andersen, P.H. and Johansen, I.S. (2011) Tuberculous Meningitis in Denmark: A Review of 50 Cases. BMC Infectious Diseases, 11, 47. http://dx.doi.org/10.1186/1471-2334-11-47

[31] Anderson, N.E., Somaratne, J., Mason, D.F., Holland, D. and Thomas, M.G. (2010) A Review of Tuberculous Meningitis at Auckland City Hospital, New Zealand. Journal of Clinical Neuroscience, 17, 1018-1022. http://dx.doi.org/10.1016/j.jocn.2010.01.007

\section{List of Abbreviations}

Tuberculosis (TB)

Central nervous system tuberculosis (CNS-TB)

Cerebrospinal Fluid (CSF)

Lumbar puncture (LP)

Mycobacterium tuberculosis (MTB)

White blood cells (WBC)

Acid-fast bacilli (AFB)

Cranial computed tomography (CT)

Magnetic resonance imaging (MRI)

Isoniazid (INH)

Intensive care unit (ICU) 